\title{
Weight loss outcomes in premenopausal versus postmenopausal women during behavioral weight loss interventions: A systematic review and meta-analysis
}

Zoe O Thomson ${ }^{1}$ APD; Jaimon T Kelly ${ }^{2}$ APD, PhD; Amanda Sainsbury ${ }^{3}$ PhD; Marina M Reeves ${ }^{1}$ AdvAPD, $\mathrm{PhD}$

${ }^{1}$ The University of Queensland, School of Public Health, Herston, Queensland, Australia

${ }^{2}$ Menzies Health Institute Queensland, Griffith University, Gold Coast, Queensland, Australia

${ }^{3}$ The University of Western Australia, Faculty of Science, School of Human Sciences, Crawley, Western Australia, Australia

Running title: Weight loss in premenopausal versus postmenopausal women

Funding: This work was supported by the National Health and Medical Research Council (NHMRC) of Australia via a Senior Research Fellowship (1135897) to AS.

\section{Corresponding author}

Zoe O Thomson

The University of Queensland School of Public Health, Level 4 Public Health Building, Herston Rd, Herston, QLD 4006.

Telephone: +61733655163

Email: z.thomson@uq.edu.au

Conflicts of interest: AS owns $50 \%$ of the shares in Zuman International Pty Ltd, which receives royalties for books she has written about adult weight management, and payments for presentations at industry conferences. She has also received presentation fees and travel reimbursements from Eli Lilly and Co, the Pharmacy Guild of Australia, Novo Nordisk, the Dietitians Association of Australia, Shoalhaven Family Medical Centres, the Pharmaceutical Society of Australia, and Metagenics, and she served on the Nestlé Health Science Optifast VLCD advisory board from 2016 to 2018. Acknowledgements: ZT is supported by a Research Training Program Scholarship. JK is supported through a Griffith University Postdoctoral Research Fellowship. AS is supported via the National Health and Medical Research Council (NHMRC) of Australia with a Senior Research Fellowship (1135897). We thank Jennifer Job for her research assistant support with the risk of bias scoring. 


\section{Key points}

Question/objective: To compare changes in weight, fat mass and lean mass in premenopausal versus postmenopausal women in weight loss trials.

Findings: Seven publications (10 interventions; $n=791$ ) were included. In meta-analyses, no statistically significant differences were observed between subgroups (premenopausal minus postmenopausal) for weight ( 0.58 [ $95 \%$ confidence interval -0.12 to 1.28$] \mathrm{kg} ; \mathrm{n}=7$ interventions), fat mass $(0.73[-0.25$ to 1.70$] \mathrm{kg} ; \mathrm{n}=6)$, or lean mass change $(-0.56[-1.48$ to 0.36$] \mathrm{kg} ; \mathrm{n}=4)$.

Meaning: Based on the limited available evidence, weight loss interventions show similar effectiveness for pre- and postmenopausal women. Subgroup analyses in additional weight loss trials and better reporting of menopausal status are needed. 


\section{Abbreviations}

BMI: body mass index

\section{Abstract (word limit: 350 words)}

Importance: Weight loss may be difficult for young women with obesity to achieve due to competing priorities (caring for children and/or full time work), limiting their ability to engage in weight loss interventions. Older or postmenopausal women may also face challenges to weight loss such as caring responsibilities and menopause. Menopausal status may reflect differences in weight loss.

Objective: This study compared changes in weight, fat mass and lean mass in premenopausal versus postmenopausal women in dietary weight loss trials.

Evidence review: We reviewed publications from January 2000 to June 2020 evaluating a weight loss intervention with a dietary component, with or without exercise, and reporting weight loss of premenopausal and postmenopausal women. Where available, data on mean change from baseline for weight, fat mass and lean mass of premenopausal and postmenopausal groups were entered into Review Manger for meta-analyses. Differences between menopausal groups were compared in subgroups of studies for intervention characteristics (diet-only versus diet and exercise; dietary modification versus meal replacement; $<24$ weeks versus $\geq 24$ weeks duration).

Findings: Seven publications (10 interventions, $n=791)$ were included; three single arm trials, two randomized controlled trials and two comparative effectiveness trials. In meta-analyses, there were no statistically significant differences between premenopausal and postmenopausal women (shown as premenopausal minus postmenopausal) for change in weight $(0.58$ [95\% confidence interval -0.12 to $1.28] \mathrm{kg}, \mathrm{n}=7$ interventions), fat mass $(0.73[-0.25$ to 1.70$] \mathrm{kg}, \mathrm{n}=6$ interventions $)$, or lean mass (-0.56[1.48 to 0.36$] \mathrm{kg}, \mathrm{n}=4$ interventions). However, a statistically significant subgroup difference was observed for fat mass change between menopausal groups (premenopausal minus postmenopausal) when comparing diet-only ( $1.28[0.23$ to 2.33$] \mathrm{kg}, \mathrm{n}=4$ interventions) versus diet and exercise interventions $(-0.09[-0.51$ to 0.32$] \mathrm{kg}, \mathrm{n}=2$ interventions $)$. No differences were shown in any other subgroups.

Conclusions and relevance: This review provides some evidence to suggest weight loss interventions may not need to be tailored to women's menopausal status. However, given the small number of studies, short intervention duration in most publications ( $\leq 6$ months) and unclear retention rates in preversus postmenopausal groups of some publications, menopausal group differences should be examined in existing and future trials where the appropriate data have been collected.

Keywords: menopause, obesity, weight loss, body composition, diet, exercise 


\section{INTRODUCTION}

More than $60 \%$ of the population in western countries are classified as having overweight or obesity ${ }^{1}$. Obesity is the leading contributor to a range of chronic diseases including cardiovascular disease, type 2 diabetes, hypertension, and certain cancers ${ }^{2-4}$. Weight loss achieved through lifestyle modification is the first line treatment for obesity ${ }^{5-7}$. Weight loss of $\geq 5 \%$ body weight has been shown to reduce the incidence and progression of many chronic diseases ${ }^{8-11}$. Weight loss may be particularly important for some population groups.

Intervening in earlier adulthood presents an opportunity to prevent the development of obesityrelated chronic diseases such as type 2 diabetes ${ }^{8}$. Despite the potential benefits of treating obesity earlier, weight loss may be difficult to achieve in earlier adulthood. For example, younger participants (aged 18-49 years) in a large weight loss program were more likely to withdraw from the study ( $<6$ months) than middle aged (50-64 years) and older ( $\geq 65$ years) participants ${ }^{12}$. Furthermore, in a large weight loss trial promoting a reduced-energy Mediterranean diet, female (compared to male) participants were 2.4 times less likely to successfully complete the program (defined as completing the program and achieving $\geq 5 \%$ weight loss $)^{13}$. In addition, each incremental year of age increased the likelihood of program completion by $1.6 \%{ }^{13}$. From qualitative research, lack of time has been cited as a prominent reason for withdrawal from an exercise program in women aged 18-30 years ${ }^{14}$, and as a barrier to participating in weight loss programs for women aged $30-40$ years ${ }^{15}$. Young women are often balancing multiple priorities such as full-time work and young children to care for, which may negatively impact on engaging in weight loss programs ${ }^{16}$.

Although younger women may face challenges in losing weight, older or postmenopausal women may also face challenges to weight loss such as caring responsibilities and biological challenges, notably menopause. The menopausal transition has been associated with reduced energy expenditure and fat oxidation ${ }^{17-19}$. Although research indicates the weight gain observed during midlife is likely due to age, not menopausal status, longitudinal studies show an accumulation of fat mass and redistribution to central adiposity during the menopausal transition ${ }^{18,20}$. The change in body composition from the menopausal transition may require women to restrict their energy intake or increase exercise to a greater extent to achieve weight loss, relative to premenopausal women. Given the change in body composition some women experience following the menopausal transition, fat mass and lean mass change from weight loss may vary by menopausal status. Therefore, menopausal status may reflect both physiological and life stage differences between younger and older women, and be reflected by differences in weight loss or body composition changes. However, it is currently unknown whether weight loss or body composition outcomes from lifestyle interventions differ between pre- and postmenopausal women. Previous reviews have focused on weight loss interventions 
for young women ${ }^{21}$ and postmenopausal women ${ }^{22}$ separately, however no review has compared the effectiveness of weight loss interventions between pre- and postmenopausal women. That is the purpose of this review. Findings from this review have the potential to inform intervention design and analyses, particularly for trials recruiting women across the lifespan. Findings may also inform current weight loss advice delivered by clinicians to individuals wanting to lose weight.

\section{METHODS}

\section{Search strategy and eligibility criteria}

This review was completed in line with the Preferred Reporting Items for Systematic Reviews and Meta-analyses (PRISMA) guidelines ${ }^{23}$. Articles published from January 2000 to June 2020 and available in English were identified in the following databases: Pubmed, Embase, Web of Science and CINAHL. The following search terms were used: ("weight loss" OR "weight reduction" OR "weight management") AND (menopaus* OR premenopaus* OR postmenopaus* OR perimenopaus*) AND (program* OR trial OR intervention). The search terms used for each database are available in Supplementary Materials Table S1. The reference lists of included publications were also manually searched for potentially relevant publications.

Publications were required to meet the following criteria to be included: (i) conducted in humans; (ii) evaluating a weight loss or weight loss maintenance intervention with a dietary component with or without exercise; (iii) conducted in adults aged $\geq 18$ years, and reporting weight loss of women from two or more menopausal groups (premenopausal, perimenopausal and/or postmenopausal). Interventions evaluating exercise only or bariatric surgery as a method of weight loss were excluded. The first author (ZOT) screened the title and abstract of all references and the full text of publications deemed potentially eligible. If eligibility was unclear from the title and abstract, the full text was reviewed. A research assistant also screened the full-text of these potentially eligible publications. Any disagreements in the eligibility of the full-text publications were resolved by discussion with a second author (MMR).

\section{Assessment of methodological quality}

The risk of bias was assessed by applying two tools - the Cochrane Collaborator's Tool (Risk of Bias 2.0) ${ }^{24}$ and the Checklist for the Appraisal of Moderators and Predictors (CHAMP) ${ }^{25}$. The Risk of Bias 2.0 tool $^{24}$ assesses the overall quality of the study and reporting in the publication, whereas the CHAMP checklist assesses the quality of moderator and subgroup analyses, which were the focus of this review ${ }^{26}$. The CHAMP checklist only includes questions specific to analyses, and recommends using a risk of bias tool to assess the overall quality of the study and reporting in the publication ${ }^{25}$. 
One domain from the Risk of Bias 2.0 tool and six items from the CHAMP checklist were unanswered, as these criteria were not relevant to the publications included in this review. The first author (ZOT) and a research assistant assessed the quality of the study and reporting in each publication. Any disagreements in scoring were resolved by discussion. For the Risk of Bias 2.0 tool, publications were assigned as either being 'high risk', 'some concerns' or 'low risk' of bias against each assessment criteria. For the CHAMP checklist, publications were assigned 'yes', 'no', 'don't know' or 'not applicable' in response to each criterion.

\section{Data extraction}

Data were extracted by one author (ZOT) and checked for accuracy by a second author (JTK). The data extracted from publications were first author family name, year, study design, intervention duration, sample size, menopausal status, BMI, intervention details, retention, and the mean and associated standard deviation (SD) for changes in weight, fat mass and lean mass (as shown in Table 1). Corresponding authors of included publications were contacted by email if further information and/or data were required for the meta-analysis. A follow-up email was sent to corresponding authors if a response was not received within two weeks of the initial request. After three email attempts with no reply, the publication was excluded from the meta-analysis.

\section{Data analysis}

Where sufficient information was available, data were pooled into Review Manager (Revman, version 5.3, Cochrane Collaboration 2014) for meta-analysis. Mean changes from baseline (with SD, 95\% confidence interval (CI) or SEM) for weight, fat mass and lean mass of pre- and postmenopausal groups were extracted and entered into Revman (by ZOT and checked by JTK). As we were interested in differences in weight loss between menopausal groups and as there was a paucity of randomized controlled trials with a non-intervention control group, only change data from intervention arms were extracted (i.e. not control arms). Some publications with multiple intervention arms reported outcomes (changes in weight, fat mass or lean mass for each menopausal group) for all interventions combined, not for each intervention. For these publications, change data from combined interventions were used. For studies that did not report change in weight, fat mass and lean mass of pre- and postmenopausal groups, but instead reported absolute mean values at baseline and end of intervention: if baseline values between the menopausal groups were similar (within $3 \mathrm{~kg}$ across each study arm), then mean weight and SD at end of intervention were used; if baseline values of any particular outcome differed between menopausal groups by $>3 \mathrm{~kg}$, mean change $(\mathrm{kg})$ in that particular outcome was calculated by subtracting the mean baseline value from the mean end of intervention value. As none of the publications included in our meta-analyses reported variability in the change data (only the variability 
in the baseline and end of intervention data), the SD for the change data were calculated in Revman from the within-group p-value for the change from baseline to end of intervention ${ }^{27}$. The overall effects for the differences in weight loss between menopausal groups were reported as mean differences between menopausal groups, with 95\% CIs, using a random effects model ${ }^{28}$. Differences in weight, lean mass or fat mass change between pre- and postmenopausal groups of at least $2 \mathrm{~kg}$ or $3 \%$ were considered meaningful regardless of statistical significance ${ }^{29}$. Statistical heterogeneity was assessed using the chi-squared test, Tau $(T)$ and the $I^{2}$ statistic and associated $\mathrm{p}$ value, and was considered low if the $\mathrm{I}^{2}$ value was $<25 \%$, moderate if the $\mathrm{I}^{2}$ value was $25-60 \%$, or high if it was $>60 \%$.

To further explore the effect of weight loss interventions by menopausal status, subgroup analyses were conducted in Revman to examine potential differences between menopausal groups according to intervention characteristics. Weighted mean differences in weight, fat mass and lean mass change between pre- and postmenopausal groups were compared in the following intervention subgroups: diet only versus diet and exercise; dietary modification versus meal replacement; and $<24$ weeks versus $\geq 24$ weeks intervention duration. Interventions were defined as 'diet only' if they did not report exercise being part of their intervention, or if they instructed participants to maintain their current level of exercise. 'Diet and exercise' interventions were those that specified promoting an increase in exercise to participants. Intervention arms were defined as 'dietary modification' if interventions encouraged participants to modify their food intake to meet macronutrient and/or energy intake goals. 'Meal replacement' interventions were those where participants were asked to use meal replacement products to replace two or more meals per day. Intervention duration was defined as the duration participants were receiving communication from intervention staff and were encouraged to follow the intervention diet.

\section{RESULTS}

The search identified 3980 potentially-eligible publications (shown in Figure 1). After removing duplicates $(n=1,600), 2,252$ titles and abstracts and 128 full-text publications were assessed for eligibility. There were 121 full text publications that were excluded based on eligibility criteria, leaving seven publications (with 10 interventions and 791 participants) for inclusion in this review.

\section{Study characteristics}

Study details of the seven publications included in this review are shown in Table 1. Six of the seven publications evaluated a weight loss intervention ${ }^{30-35}$. One publication included an initial 6month weight loss phase followed by a 6-month weight maintenance phase; however in this review we extracted the data available in the publication which pertains to the 6-month weight loss phase only ${ }^{36}$. 
Sample sizes ranged from 70 participants ${ }^{30}$ to 242 participants ${ }^{35}$. Six publications recruited exclusively pre- and postmenopausal women (excluding perimenopausal women) [28-32], and one publication conducted secondary data analysis of pre- and postmenopausal participants from a larger publication recruiting men and women aged 30-70 years (excluding data from perimenopausal women) [33]. Intervention durations ranged from 3 months ${ }^{30,32,34}$ to 24 months ${ }^{35}$. The seven publications included three single-arm trials ${ }^{31,32,34}$, two randomized controlled trials ${ }^{33,36}$, and two comparative effectiveness trials (where participants were randomized to one of two or more intervention groups) ${ }^{30}$, 35 .

Four of the seven publications (six interventions) delivered the intervention via face-to-face contact $^{31,33,35,36}$, using both individual and group sessions ${ }^{31,35}$, individual sessions only ${ }^{36}$, or group sessions only ${ }^{33}$. The mode of intervention delivery in the remaining three publications (four interventions) was not reported ${ }^{30,32,34}$. Three publications (six interventions) evaluated interventions involving dietary modification only ${ }^{30,33,36}$, while the remaining four publications (four interventions) promoted modifications to both diet and exercise ${ }^{31,32,34,35}$. Three interventions utilized meal replacement diets $31,33,34$.

\section{Risk of Bias}

Assessment of risk of bias is shown in Table 2. The overall quality of included publications was mixed. Common limitations with the overall study quality assessed by the Risk of Bias 2.0 tool included bias arising from the randomization process, and bias due to deviations from the intended interventions. For assessing the analysis of menopausal groups using the CHAMP checklist, few publications met criteria for a priori specification of subgroup analyses and power to conduct subgroup analyses. Three of the seven publications did not meet criteria for missing outcome data; this was primarily due to high attrition rates and use of complete-case analysis. Further, only one publication reported the attrition rates by menopausal subgroup, showing $25 \%$ attrition in the premenopausal group compared to $17 \%$ in the postmenopausal group at 24 months ${ }^{35}$. For menopausal status, four of the seven publications adequately assessed postmenopausal status as being 12 months of amenorrhea ${ }^{32-35}$. However, none of the seven publications reported adequate information about how premenopausal status was assessed.

\section{Weight loss comparison by menopausal status}

All seven publications included in this review reported weight loss of both pre- and postmenopausal participants ${ }^{30-36}$. Six publications reported results for individual intervention arms ${ }^{30-}$ 32, 34, 36. One publication with four interventions grouped data from these four interventions ${ }^{35}$. Metaanalyses were conducted with seven interventions (from five publications) to investigate the 
differences in change in weight between pre- and postmenopausal women receiving the same intervention in each publication (i.e. the 'comparison' being menopausal status rather than an intervention per se) (see Figure 2) ${ }^{30,31,33-35}$. Meta-analysis showed there was a non-significant difference in absolute weight loss between groups (weight loss in premenopausal minus weight loss in postmenopausal women) (0.58 [95\% CI -0.12 to 1.28$\left.] \mathrm{kg}, I^{2}=20 \%\right)$. Of the 10 interventions, only three reported a clinically meaningful $(2 \mathrm{~kg}$ or $3 \%$ ) difference in weight loss between pre- and postmenopausal groups, with greater weight loss in postmenopausal groups in these three interventions 34,36 .

Forest plots for subgroup analyses are shown in Supplementary Materials Figure S1 and are summarized in Table 3. No statistically significant subgroup differences in the pooled effects (preminus postmenopausal) for weight change were observed between any of the intervention characteristic subgroups: diet only interventions versus diet and exercise interventions; dietary modification interventions versus meal replacement interventions, and interventions with a duration of $<24$ weeks versus those $\geq 24$ weeks.

Two (of seven) publications included in this review were unable to be included in the metaanalysis. Consistent with results from the meta-analysis, Nakamura et al. reported a non-significant difference in mean BMI change between menopausal groups $\left(-1.2 \mathrm{~kg} / \mathrm{m}^{2}\right.$ for pre- versus $-1.1 \mathrm{~kg} / \mathrm{m}^{2}$ for postmenopausal woman; 95\% CI not reported) ${ }^{32}$. In contrast to results from our meta-analysis, Barnosky et al. reported significantly smaller weight loss for premenopausal women compared to postmenopausal women in both interventions investigated in their study (premenopausal $-6.0 \pm 1.1 \%$ Of initial body weight and $-6.0 \pm 1 \%$ versus postmenopausal $-11.2 \pm 2.3 \%$ and $-11.6 \pm 3.7 \%$ ). However, the sample size for the menopausal groups was unclear and potentially small, as the total sample sizes for the two interventions were $n=25$ and $n=29$ respectively.

\section{Fat mass and lean mass change by menopausal status}

Six of the seven publications included in this review also reported change in body composition (fat mass and/or lean mass) by menopausal status (shown in Supplementary Materials Table S2) ${ }^{30,31,}$ 33-36. Body composition was measured by dual-energy $x$-ray absorptiometry in four of these six publications ${ }^{30,33,35,36}$, by bioelectrical impedance in one publication ${ }^{34}$, or by air displacement plethysmography in the other of these six publications ${ }^{31}$.

For fat mass change, a meta-analysis of five publications ( 7 interventions) showed there was a non-significant difference in fat mass loss between pre- and postmenopausal groups (premenopausal minus postmenopausal: 0.73 [-0.25 to 1.70$] \mathrm{kg}, I^{2}=41 \%$ ) (refer to Figure 2). The difference in fat mass change between pre- and postmenopausal women was also compared according to intervention characteristics (Supplementary Materials Figure S2 and Table 3). A statistically significant subgroup 
difference was observed for change in fat mass between the diet only versus diet and exercise interventions ( $\mathrm{p}=0.02, I^{2}=82.4 \%, \mathrm{n}=$ six interventions). No statistically significant subgroup differences (pre- minus postmenopausal) in the pooled effects for fat mass change were observed for: dietary modification interventions versus meal replacement interventions, and interventions with a duration of $<24$ weeks versus those $\geq 24$ weeks.

Four of the seven publications included in this review reported change in lean mass $30,31,35,36$, with three of these four publications (4 interventions) being included in the meta-analysis ${ }^{30,31,35}$. No significant difference in lean mass change between menopausal groups was observed (-0.56 [-1.48 to $0.36] \mathrm{kg}, I^{2}=83 \%$ ) (shown in Figure 2). No statistically significant subgroup differences (pre- minus postmenopausal) in the pooled effects for lean mass change were observed between any of the intervention characteristic subgroups.

\section{Discussion}

Premenopausal and postmenopausal women may face separate challenges to losing excess weight. However, to our knowledge, the evidence comparing weight loss and body composition changes between pre- and postmenopausal women has not previously been systematically synthesized in a review. This review compared changes in weight, fat mass and lean mass between pre- and postmenopausal women in weight loss trials. Findings from the publications included in our metaanalyses showed similar change in these three parameters between pre- and postmenopausal women in weight loss interventions. From these findings, albeit at there is little available data, there is a lack of evidence to suggest interventions should be tailored to women's menopausal status.

Results from this systematic review and meta-analysis extend results from a previous systematic review, which showed that in a majority of studies ( $\mathrm{n}=9$ of 11 ), age was not a determinant of maintenance of weight loss ${ }^{37}$. That review of determinants of weight loss maintenance may not be directly comparable to the current review, as they included studies of men and women ( $\mathrm{n}=8$ of 11$)$ and investigated weight loss maintenance, not weight loss ${ }^{37}$. Specific to weight loss, a study of women $(n=100)$ undergoing a weight loss program promoting a reduced-energy diet and exercise reported no statistically-significant difference in the mean age between those that achieved no weight loss or weight gain versus those that achieved weight losses of $\geq 9 \%$ of their initial body weight ( 45.9 years versus 42.4 years) ${ }^{38}$. Conversely, a large difference in mean weight loss was observed across age groups in a weight loss trial promoting a reduced-energy diet and exercise in 692 women following treatment for breast cancer ${ }^{39}$. At the end of the 24-month intervention in that study, mean weight loss was $0.0 \%$ in women aged $30-44$ years, $2.1 \%$ in women aged $44-54$ years, and $5.2 \%$ in women aged 55 82 years ${ }^{39}$. This difference in weight loss across age groups may be unique to populations of women 
with breast cancer. Women diagnosed with premenopausal breast cancer commonly undergo an accelerated and premature menopause associated with a reduction in lean mass and resting energy expenditure ${ }^{40}$. A reduction in energy expenditure may explain the lack of weight loss that was observed in younger women in that weight loss trial ${ }^{39}$. It is unclear if the results observed in that study may be unique to the breast cancer population and not generalizable, with the results from the current review suggesting similar weight loss across menopausal groups.

Of the publications included in the present systematic review, one publication, which could not be included in the meta-analysis, implemented two weight loss interventions and showed large differences in weight loss between pre- and postmenopausal women (premenopausal $-6.0 \pm 1.1 \%$ Of initial body weight and $-6.0 \pm 1 \%$ versus postmenopausal $-11.2 \pm 2.3 \%$ and $-11.6 \pm 3.7 \%)^{36}$. These differences were not explained by differences in physical activity (i.e. similar steps/day between menopausal groups), and no data were reported on energy intake. No other studies that observed differences in weight loss between pre- and postmenopausal women in this review reported on potentially underpinning behaviors (diet and exercise). Examining diet and exercise differences between women of different menopausal status in future research may help to explain variability between publications and groups of participants.

This systematic review has a number of strengths and limitations. Strengths include this being the first review to compare changes in weight and body composition in response to a weight loss intervention between pre- and postmenopausal women. Additionally, this review adhered to PRISMA guidelines in its conduct and reporting. We also chose to utilize two risk of bias tools to assess both the overall study quality and subgroup analyses. However, this review has important limitations to consider. Firstly, this review is limited by the pooling of publications with small sample sizes and heterogeneous designs. . Secondly, risk of bias scoring identified common limitations across publications, specifically that publications did not specify analyses in separate groups of women (preor postmenopausal) a priori, and were not powered to conduct analyses in these two groups of women. Third, all publications included in this review either did not adequately assess, or lacked sufficient detail on the method of assessing, premenopausal and perimenopausal status. This poor assessment may have led to some inclusion of perimenopausal women in the premenopausal groups. Therefore, consistency in assessing pre- versus perimenopausal status is warranted in future, and can be standardized by utilizing criteria such as those listed in the Stage of Reproductive Aging Workshop +10 (STRAW+10) tool ${ }^{41}$. This tool provides detailed information on staging for reproductive aging in women. It involves asking women to self-report bleeding pattern and frequency. Its use in future would improve the assessment of menopausal status, particularly differentiating between pre- and perimenopausal women. A further limitation of this review is that Most of the included publications 
evaluated short-term weight loss interventions ranging from 12-26 weeks. Thus generalizability to longer-term interventions ( $>6$ months) and differences in weight regain are limited. Moreover, although the review was open to weight loss and weight loss maintenance trials, all included publications reported results of initial weight loss.

\section{Conclusion}

While the findings from the meta-analysis suggest that weight loss interventions are similarly effective for pre- and postmenopausal women, the available evidence on which this is based is limited and thus is inconclusive. Further research is needed to determine conclusively, whether differences in weight loss between menopausal groups exist and whether interventions should be tailored. Existing trials and future trials should determine menopausal status using criteria such as STRAW +10 to enable data to be pooled and subgroup differences to be examined. 


\section{REFERENCES}

1. Organisation for Economic Co-operation and Development. OECD Obesity update. OECD; 2014.

2. Calle EE, Kaaks R. Overweight, obesity and cancer: epidemiological evidence and proposed mechanisms. Nat Rev Cancer. [10.1038/nrc1408]. 2004;4(8):579-91.

3. Rabkin SW, Chen Y, Leiter L, Liu L, Reeder BA. Risk factor correlates of body mass index. Canadian Heart Health Surveys Research Group. CMAJ : Canadian Medical Association journal = journal de l'Association medicale canadienne. 1997;157 Suppl 1:S26-31.

4. Pi-Sunyer FX. Medical hazards of obesity. Annals of internal medicine. 1993;119(7 Pt 2):655-

60.

5. National Health and Medical Research Council. Clinical practice guidelines for the

management of overweight and obesity in adults, adolescents and children in Australia. In: Health Do, editor. Canberra: Australian Government 2013.

6. Jensen MD, Ryan DH, Apovian CM, et al. 2013 AHA/ACC/TOS Guideline for the Management of Overweight and Obesity in Adults. A Report of the American College of Cardiology/American Heart Association Task Force on Practice Guidelines and The Obesity Society. 2013.

7. National Institute for Health and Care Excellence. Weight management: lifestyle services for overweight or obese adults. UK: NICE; 2014.

8. Hamman RF, Wing RR, Edelstein SL, et al. Effect of weight loss with lifestyle intervention on risk of diabetes. Diabetes care. 2006;29(9):2102-7.

9. Phelan S, Kanaya AM, Subak LL, et al. Weight loss prevents urinary incontinence in women with type 2 diabetes: results from the Look AHEAD trial. The Journal of urology. 2012;187(3):939-44.

10. Magkos F, Fraterrigo G, Yoshino J, et al. Effects of moderate and subsequent progressive weight loss on metabolic function and adipose tissue biology in humans with obesity. Cell metabolism. 2016;23(4):591-601.

11. Chlebowski RT, Luo J, Anderson GL, et al. Weight loss and breast cancer incidence in postmenopausal women. Cancer. 2019;125(2):205-12.

12. Jiandani D, Wharton S, Rotondi MA, Ardern CI, Kuk JL. Predictors of early attrition and successful weight loss in patients attending an obesity management program. BMC obesity. 2016;3:14. 13. Bautista-Castaño I, Molina-Cabrillana J, Montoya-Alonso JA, Serra-Majem L. Variables predictive of adherence to diet and physical activity recommendations in the treatment of obesity and overweight, in a group of Spanish subjects. International Journal of Obesity. 2004;28(5):697-705.

14. Arikawa AY, O'Dougherty M, Kaufman BC, Schmitz KH, Kurzer MS. Attrition and adherence of young women to aerobic exercise: lessons from the WISER study. Contemporary clinical trials. 2012;33(2):298-301.

15. Crino ND, Parker HM, Gifford JA, et al. Recruiting young women to weight management programs: Barriers and enablers. Nutrition \& Dietetics. 2019;76(4):392-8.

16. Klem ML, Viteri JE, Wing RR. Primary prevention of weight gain for women aged 25-34: the acceptability of treatment formats. International Journal of Obesity. 2000;24(2):219-25.

17. Macdonald HM, New SA, Campbell MK, Reid DM. Longitudinal changes in weight in perimenopausal and early postmenopausal women: effects of dietary energy intake, energy expenditure, dietary calcium intake and hormone replacement therapy. Int J Obes Relat Metab Disord. 2003;27(6):669-76.

18. Lovejoy JC, Champagne CM, de Jonge L, Xie H, Smith SR. Increased visceral fat and decreased energy expenditure during the menopausal transition. International journal of obesity (2005). 2008;32(6):949-58.

19. Duval K, Prud'homme D, Rabasa-Lhoret R, et al. Effects of the menopausal transition on energy expenditure: a MONET Group Study. European journal of clinical nutrition. 2013;67(4):40711. 
20. Lee CG, Carr MC, Murdoch SJ, et al. Adipokines, inflammation, and visceral adiposity across the menopausal transition: a prospective study. J Clin Endocrinol Metab. 2009;94(4):1104-10.

21. Hutchesson MJ, Hulst J, Collins CE. Weight management interventions targeting young women: a systematic review. Journal of the Academy of Nutrition and Dietetics. 2013;113(6):795-802. 22. Cheng CC, Hsu CY, Liu JF. Effects of dietary and exercise intervention on weight loss and body composition in obese postmenopausal women: a systematic review and meta-analysis. Menopause (New York, NY). 2018;25(7):772-82.

23. Moher D, Shamseer L, Clarke M, et al. Preferred reporting items for systematic review and meta-analysis protocols (PRISMA-P) 2015 statement. Systematic Reviews. 2015;4(1):1.

24. Higgins J, Sterne J, Savović J, et al. A revised tool for assessing risk of bias in randomized trials. Cochrane Database of Systematic Reviews. 2016;10:29-31.

25. van Hoorn R, Tummers M, Booth A, et al. The development of CHAMP: a checklist for the appraisal of moderators and predictors. BMC medical research methodology. 2017;17(1):173.

26. Wu T, Gao X, Chen M, Van Dam RM. Long-term effectiveness of diet-plus-exercise interventions vs. diet-only interventions for weight loss: a meta-analysis. Obesity Reviews. 2009;10(3):313-23.

27. Wiebe N, Vandermeer B, Platt RW, Klassen TP, Moher D, Barrowman NJ. A systematic review identifies a lack of standardization in methods for handling missing variance data. Journal of clinical epidemiology. 2006;59(4):342-53.

28. DerSimonian R, Laird N. Meta-analysis in clinical trials. Control Clin Trials. 1986;7(3):177-

88.

29. Cefalu WT, Bray GA, Home PD, et al. Advances in the Science, Treatment, and Prevention of the Disease of Obesity: Reflections From a \&lt;em\&gt;Diabetes Care\&lt;/em\&gt; Editors' Expert Forum. Diabetes care. 2015;38(8):1567.

30. Clifton PM, Noakes M, Keogh JB. Very low-fat (12\%) and high monounsaturated fat (35\%) diets do not differentially affect abdominal fat loss in overweight, nondiabetic women. Journal of Nutrition. 2004;134(7):1741-5.

31. Deibert P, Konig D, Vitolins MZ, et al. Effect of a weight loss intervention on anthropometric measures and metabolic risk factors in pre- versus postmenopausal women. Nutrition Journal. 2007;6:1-7.

32. Nakamura M, Tanaka M, Kinukawa N, et al. Association between basal serum and leptin levels and changes in abdominal fat distribution during weight loss. Journal of atherosclerosis and thrombosis. 2000;6(1):28-32.

33. Shapses SA, Heshka S, Heymsfield SB. Effect of calcium supplementation on weight and fat loss in women. Journal of Clinical Endocrinology \& Metabolism. 2004;89(2):632-7.

34. Shigematsu R, Okura T, Kumagai S, et al. Cutoff and target values for intra-abdominal fat area for prevention of metabolic disorders in pre- and post-menopausal obese women before and after weight reduction. Circulation Journal. 2006;70(1):110-4.

35. Tirosh A, de Souza RJ, Sacks F, Bray GA, Smith SR, LeBoff MS. Sex Differences in the Effects of Weight Loss Diets on Bone Mineral Density and Body Composition: POUNDS LOST Trial. Journal of Clinical Endocrinology \& Metabolism. 2015;100(6):2463-71.

36. Barnosky A, Kroeger CM, Trepanowski JF, et al. Effect of alternate day fasting on markers of bone metabolism: An exploratory analysis of a 6-month randomized controlled trial. Nutrition and healthy aging. 2017;4(3):255-63.

37. Varkevisser RDM, van Stralen MM, Kroeze W, Ket JCF, Steenhuis IHM. Determinants of weight loss maintenance: a systematic review. Obesity reviews : an official journal of the International Association for the Study of Obesity. 2019;20(2):171-211.

38. Annesi JJ, Whitaker AC. Psychological factors discriminating between successful and unsuccessful weight loss in a behavioral exercise and nutrition education treatment. International journal of behavioral medicine. 2010;17(3):168-75.

39. Rock CL, Flatt SW, Byers TE, et al. Results of the exercise and nutrition to enhance recovery and good health for you (ENERGY) trial: a behavioral weight loss intervention in overweight or obese 
breast cancer survivors. Journal of clinical oncology : official journal of the American Society of Clinical Oncology. 2015;33(28):3169-76.

40. Gordon AM, Hurwitz S, Shapiro CL, LeBoff MS. Premature ovarian failure and body composition changes with adjuvant chemotherapy for breast cancer. Menopause (New York, NY). 2011;18(11):1244-8.

41. Harlow SD, Gass M, Hall JE, et al. Executive summary of the Stages of Reproductive Aging Workshop + 10: addressing the unfinished agenda of staging reproductive aging. Menopause (New York, NY). 2012;19(4):387-95. 


\section{Supplemental Digital Content}

Supplemental Digital Content 1. Preferred Reporting Items for Systematic Reviews and Meta-analyses flow chart.

Supplemental Digital Content 2. Table of weight loss trials comparing weight loss of pre- and postmenopausal participants.doc

Supplemental Digital Content 3. Table of risk of bias assessment

Supplemental Digital Content 4. Forest plot comparing absolute weight, fat mass and lean mass change in preand postmenopausal women

Supplemental Digital Content 4. Table summarizing subgroup analysis results for changes in weight, fat mass and lean mass in premenopausal women minus corresponding measures on postmenopausal women.doc

Supplemental Digital Content 5. Table of search terms used in the development of the search strategy

Supplemental Digital Content 6 . Table of change in weight and body composition of pre- and postmenopausal participants in weight loss interventions

Supplemental Digital Content 7. Forest plots comparing absolute weight change in premenopausal and postmenopausal women by intervention characteristics

Supplemental Digital Content 8. Forest plots comparing fat mass change in premenopausal and postmenopausal women by intervention characteristics

Supplemental Digital Content 9. Forest plots comparing lean mass change in premenopausal and postmenopausal women by intervention characteristics 